\title{
WIRE CHAMBER REQUIREMENTS AND TRACKING SMMULATION STUDIES FOR TRACKING SYSTEMS AT THE SUPERCONDUCTING SUPER COLLIDER*
}

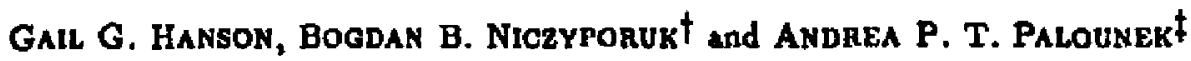
Stanford Lineer Accelenator Center, Stanford University,

Stanford, Califormia 9/509, USA

Limitatione placed on wire chambers by radiation damage and rate require ments in the SSC environment are reviewed. Ponible conceptual denigne for wire chamber tracking aystems which meet these requirements are diectused. Computer simulation studies of tracling in such systems are presented. Simulations of events from interesting physics at the SSC, including hits from minimum bias background events, are examined. Results of eome preliminary pattern recognition studies are given. Such computer simulation studies are secessary to determine the fensibility of wire chamber tracking systems for complex events in a high-rate environment wich as the SSC.

\footnotetext{
Work eupported by the Department of Energy, contrect DE-AC03-76SF00515.

$\dagger$ Permanent Addres: CEBAF, Newport Newr, Virginia 23606.

† Present Address: Lawrence Berkeley Laboratory, Berkeley, Calitornia 94720.
}

Presented at the Wire Chamber Conference, Vienna, Austria, February 19-17, 1989 


\section{INTRODUCTION}

Tracking at the SSC at the full design luminosity is expected to be a diffcult problem. The limitations imposed by rates and radiation damage are severe. However, the dominant constraint is the combination of occupancy and double-hit resolution. Single events from new physics at the SSC bave many (several hundred) charged particle tracka and are further complicated by curling tracks in a magnetic field, photon conversions, hits from events from out-of-time bunch crossings, and multiple interactions within the same bunch crossing [1]. It has not been established how well one can find tracks in complex SSC events. These problems can be addreased through compulet simulation atudien.

A large solenoid detector based on more-or-lese "conventional" lechnology was diecussed at the 1987 Berkeley Workhop [2]. The central tracking oystem for thi: detector was envisioned to be huilt of superlayers consisting of layers of straw tubes. With such a design it should be possible to identify local track segmento with a high degree of redundancy even in complex SSC events while at the same time rejecting hits from background. We report bere on computer simulation studies of tracking in such a detector.

\section{WIRE CHAMBER REQUIREMENTS}

\subsection{The SSC Environment}

$L$ ite deaign luminosity, $\mathcal{L}$, of the SSC is $10^{33} \mathrm{~cm}^{-2},-1$ with $\&$ energy of $40 \mathrm{TeV}$ in the center of mass. The inelastic cross section, $\sigma$, at $40 \mathrm{TeV}$ is expected to be about $100 \mathrm{mb}$, whicls gives $10^{6}$ interactions per second at the design iuminos:ty. The bunch separation is $4.8 \mathrm{~m}$, so the time between bunch crossings, $t_{B}$, is $16 \mathrm{I}$, 
which leads to an average number of inleractions per bunch crowing, $n$, of 1.6 at the design luminosity. Moot of these interactions are minimum bias events or low-pr hard scattering proceses in which particle production is expected to be uniform in rapidity with an average charged particle multiplicily per unit of rapidity, $n_{e}$, of 7.5 over the pecudorapidity range $|\eta|<6$ [3]. Figure 1 (from ref. [3]) shows the roulting charged particle fux and annual radiation does as a function of perpendicular distanoe from the beum for etandand SSC operating conditions.

\subsection{Rates and Radiation Damige}

Radiation damage and rate limitations impowe revere conntraints on charged particle tracking detectors at the SSC, as decribed in everal references $[1,4]$. These constraints are aummaried here since they are neocanry conaiderations for the deaign of any SSC tracting ayatem.

A trecking aystem for the SSC is anumed to be made up of wires running (nerily) paralled to the beam line The width, $w$, of the cell is asoumed to be equal to the height, $h$, and the drift distance, $d$, in half the cell width. The ionization rate, $a$, in the gas is assumed to be 100 dectrons/cm. The gas gain, $G$, is asumed to be $2 \times 10^{4}$.

The flux of particles per unit length ( 9 ) of wire in a cell at radius $r$ is given by

$$
\frac{d n}{d \ell d t}=\frac{n_{c} t \sigma \sigma \mathcal{L} \sin \theta}{2 \pi r^{2}}
$$

where is the angle relative to the beam direction. The ionization produced by a charged particle at angle $\theta$ is ha/sin $\theta$, so the ionization per unit leagth of wire 
is independent of $\theta$. Thus the current draw per wire, $I$, for a layer of wires of length $L$ at radius $r$ is given by

$$
J=\frac{n_{e} v \operatorname{hocGegL}}{2 \pi r^{2}}
$$

where $e$ is the electron charge. A layer of $4 \mathrm{~mm}$ wide cells at a radius of $50 \mathrm{~cm}$ covering $|\mathrm{h}|<1.5(L=213 \mathrm{~cm})$ will draw $0.52 \mu \mathrm{A} /$ wire. The limit of acceptable curreat dran before breakdown will oocur is about $1 \mu \mathrm{A} /$ wire.

Wire chamber lifetimes are measured in deparited charge per unit length of wire before a decreane in gain occurs due to the buildup of material on the wires. For the above example, the collected charge over a chamber litetime of five years $\left(5 \times 10^{7}\right.$ s) would be $0.12 \mathrm{C} / \mathrm{cm}$. Chamber lifetimes of $1.0 \mathrm{C} / \mathrm{cm}$ have been measurad under very clean laboratory conditions [5], but for the purpasea of a renlistic experiment, it is probably beat to arume a chamber lifetime sbout an order of magnitude leas than this.

Changea in gain for wire chamber have been observed at the level of $10^{\text {s }}$ particles/cm-a at a gas gain of $\sim 4 \times 10^{5}$ due to apace charge buildup [6]. The particle fux is given by eq. (1). For the above example, the fux would be $1.9 \times 10^{4}$ particles/cm-s at $\theta=90^{\circ}$ where the flux is maximum. Since the gas gain must be much amaller than $4 \times 10^{5}$ because of current draw and lifetime considerations, space charge should not be an important limitation.

The hit rale per wre, $R$, for SSC central tracking chambers is quite large and is given by

$$
R=\frac{n_{c} \eta_{\max } \sigma \mathcal{L}_{v}}{\pi r}
$$


for chambers covering $|\eta|<\eta_{\text {max }}$. For the above example, the hit rate per wire would be 2.9 Mhz. Existing electronics can probably handle rateg of $\sim 10 \mathrm{Mhz}$.

A very serious limitation for tracking ayatems at the SSC is nccupancy. Since the time between bunch crossinge at the SSC is shorter than the resolving time of a typical drift chamber cell, the cell is sensitive to several bunch crossings. The occupancy, $O$, ia given by

$$
O=\frac{2 n_{c} \eta_{\max } n_{l} n_{B} d}{\pi r}
$$

where $n_{B}$ is the number of bunch crossinga during the resolving time of the cell. $n_{B}$ is given by

$$
n_{B}=1+\operatorname{int}\left(t_{R} / t_{B}\right)\left[2-t_{B} / t_{R}-\left(t_{B} / t_{R}\right) \operatorname{int}\left(t_{R} / t_{B}\right)\right] \text {, }
$$

where $t_{R}$ is the resolving time of the cell, $d / v_{D}$, for drif velocity $v_{D}$, and int $(x)$ is the largest integer $\leq x$. Actually, $n_{B}$ is very close to $t_{R} / t_{B}=d /\left(v_{D} t_{B}\right)$. A $4 \mathrm{~mm}$ wide cell ( $2 \mathrm{~mm}$ drift) has a resolving time of $\mathbf{4 0} \mathrm{na}$ for a typical drift velocity of $50 \mu \mathrm{m} / \mathrm{as}$ and is therefore șensitive to 2.6 bunch croseings. A layer of such cells at a radius of $50 \mathrm{~cm}$ and covering a rapidity range $|\eta|<1.5$ would have an occupancy of $12 \%$ per cell. It is guessed that an occupancy of $\sim 10 \%$ is reasonable, but a realistic answer depends on the effects on pattern recognition and track finding, which are diacussed in more detail in sec. 3. The real limilation to ocrupancy is duc to the double-hit resolution because of the loss of informalion.

The rates given above are based only on particles produced in an interaction and muat be increased by a factor of 2-4 because of curling tracks in a magnetic 
ficld, converted photons, and albedo particlea leaking out of the front face of the calorimeter. Regardless of pattern recognition considerations, the effects on current draw and chamber lifetime muat be carefully considered in the deaign of any SSC tracking system based on wire chambers.

\subsection{Tracking Syotem Considerations}

A large colesoid detector baned on mort-or-bea "conventional" technology was discussed at the 1987 Berkeley Worksbop [2]. Calorimetry and tracking were located inside a large duperconducting solenoid with 2 Tela field. A schematic view of the Large Solenoid Detector is shown in fig. 2. An examination of the requirments for momentum resolution based on the phynics led to the criterion that the sign of the charge for electrons should be meanred for PT $\leq 0.5-1.0 \mathrm{TeV} / \mathrm{c}$. The momentum resolution is given by [7]

$$
\frac{\sigma_{q \tau}}{P \tau^{2}}=\sqrt{\frac{720}{1+5 / N}}\left(\frac{\sigma_{x}}{0.3 q B D^{2} \sqrt{N}}\right) \text {, }
$$

where $p r$ is the transverse momentum of the particle in $\mathrm{GeV} / c, q$ is the charge in units of the electron charge, $\sigma_{x}$ is the spatial resolution in $\mathrm{m}, \boldsymbol{B}$ is the magnetic field in Tesla, $D$ is the track length in $m_{3}$ and $N$ is the number of menomrements, assumed to be equally spaced.

The tracking deteclor design for the Large Solenoid Detector was divided into central tracking $(|\eta| \leqslant 1.2)$ and intermediate tracking $(1.2 \leqslant|\eta|<2.5)$. The central tracking system was assumed to be built of straw tubes of radii from 2 to $3.5 \mathrm{~mm}$ parallel or nearly parallel to the beam direction. There are many advantages to a straw tube design for tracking at the SSC, as discussed in ref. [1]. 
The straws are made of aluminized polyester film (Mylar) or polycarbonate (Lexan) with wall thickneases of about $30 \mathrm{~mm}$ and are assumed to be at alnospheric pressure. Eight layers of straws are glued together to form superlayers. Within each superlayer the layers are staggered by half the cell width in order to allow hits from out-of-time bunch crossings to be rejected and resolve left-right ambiguities, as illustrated in fig. 3. By dividing the chamber into eight-straw-thick superlayers locally identifiable track segments can be obtained with a bigh level of redundancy. The track segments can then be linked to form tracks. Every other superlayer is small-angle sterco $\left(\sim 3^{\circ}\right)$ in order to measure the coordinate along the wirc. Azimuthal cathode pads or strips, included on the outer surfaces of the superlayers, will be useful for bunch assignment and reducing stereo ambiguities. The central tracking system extends radially from $40 \mathrm{~cm}$ to $160 \mathrm{~cm}$ with 15 superlayers in all. Assuming a spatial resolution of $150 \mu \mathrm{m}$, the momentum resolution which can be obtained with such a system is $0.54 \mathrm{pT}_{T}(\mathrm{TeV} / \mathrm{c})$ using only wires at radii larger than $50 \mathrm{~cm}$. If the particles are constrained to come from the interaction region, the momentum resolution would improve to $0.26 \mathrm{pT}$. The total number of cells is 122,368. The total number of radiation lengths is $8 \%$ for a particle iraversing the central tracking chambers at $90^{\circ}$. The Large Solenoid Detector central tracking system geometry is summarized in table 1.

In order to provide momentum measurement for $1.2 \leqslant|\eta|<2.5$, tu: Large Solenoid Detector included tracking in the intermediate region to take over where the central tracking ends. Two options were considered: planes of narallel wires and radial chambers. The options for intermediate tracking have not yet been worked out in as much detail as the central tracking. 
The central and intermediate tracking systems for the Large Solenoid Delector are shown in fig. $4(\mathrm{a})$, and the momentum resolution as a function of polar angle and rapidity is shown in fig. $4(\mathrm{~b})$.

\section{TRACKING SIMULATION}

\subsection{Simulation of a Central Tracking System for the SSC}

The simulation of an SSC central tracking system design was based on that for the Large Solenoid Detector [2], described in sec. 2.3, although it is quite general and can be used for any syslem of cylindritally oriented sensing elements. All parameters of the detector, such as number of superlayers, number of layers in each superlayer, minimum and maximum radius and length of each superlayer, and azimuthal spacing between sense wires can be specified independently. The parameters used are as shown in table 1 , except that we included only the outer 13 superlayers. We used a solenoidal magnetic field of 2 Tesla. The spatial resolution was taken to be $150 \mu \mathrm{m}$. So far, we have simulated only axial wires, that is, wires parallel to the cylinder axis.

We used ISA.JET [8] to generate events from interesting physiss processes, such as high-p $p_{T}$ two-jet events or Iliggs boson production, and from inelastic acattering background, for which we used minimum bizs events. We used the GEANT3 [9] general-purpose detector simulation package running on the SLAC IBM 3081 to simulate the interactions of the particles with the delector.

Using GEANT, the particles interact in the $8 \%$ of a radiation length of matcrial due to straw tube walls, wires, and gas (the material was assumed to be 
distributed uniformly throughout the tracking volume), including photon conversion and multiple Coulomb scattering. The digitizations consist of a wire number and a drift time, calculated from the distance of closest approach of a track to a wire using a drift velocity of $50 \mu \mathrm{m} / \mathrm{ns}$, for each track in each layer. Background from inelastic scatterings in the same and out-of-time bunch crossings is included by superimposing the digitizations from minimum bias events. The number of bunch crossings is determined by the resolving time of the atraw tube cell. At each bunch crossing the number of events to be included is determined from a Poisson distribution with a mean of 1.6 interactions per bunch crousing. Drift times from background events are then corrected for the time difference between the bunch crosoing of the background event and the bunch croseing of the event of interest. The double hit reeolution is equal to the cell width, that is, only the carlieat hit on a wire is kept. The aimulation program is described in more detail in ref. [9].

\subsection{Results of the Simulation}

We used the simulation deacribed above to study tracking in SSC events. First. we examined high-pr (pr $>1 \mathrm{TeV} / \mathrm{c}$ ) two-jet events. Figure $5(a)$ shows such an event in the detector described in sec. 2.3. Figure 5(b) shows an enlargement of the same event in the outer two superlayers in the ares of the dense jet. Figure $5(c)$ show the earlieat hite in the cells for the tracks shown in fig. 5(b). Hits from background events and converted photons are not shown in fig. 5 .

Although we have not yet quantified theae observations with high-statistics studies, we nole: 
1. Although these events have very denge jets which seem at first lo be imposgjble to resolve, when viewed on the scale of the wire spacings most of the hits appear to lie on identifiable tracks with a 2-Tesla magnetic field, particularly in the outer guperlayers.

2. Bight layers in a superlayer is probably close to the optimum number because two tracks which are as close as the wire apacings produce hits only on every other layer because of the staggering. Some of these bits may be lost due to nearby curling tracks or background hits. Three tracke within the wire spacing distance would not be revolvable.

3. Although a 2-Teala magnetic field produces curling tracks which obscure the high-pr tracks to some extent, particularly in the inner superbyer, the effect in the outer nuperlayers is to spread out the tracke and, of course, remove the low-pr track from consideration.

We next turned our attention to events from Higzs bown production, $p P \rightarrow H X$, with the Higgs decaying to $Z^{0} Z^{0}$ and both $Z^{0} \cdot$ decaying to $e^{+} e^{-}$ or $\mu^{+} \mu^{-}$. We used a Higg mass of 400 GeV/c. Such events allowed us to focus on the measurement of the high-pr particles from the Higg decay. Leptons from heavy Hists decay typically have PT $>20 \mathrm{GeV} / \mathrm{c}$. For these events we ured the full simulation as described in the previous Section. An example of a Higgs event in the simulated central tracking oystem is shown in fig. 6 . We generated $\sim 200$ such events.

The fully-simulated events, including adding digitizations from minimum bias background events and removing digitizations within the double-hit resolution, 
had 12,000-30,000 digitizations, as shown in fig. $7(\mathrm{a})$. The fraction of digilizations from the minimum bias background events is shown in fiz. $7(b)$. On the average $57 \%$ of the digitizations were due to background events. For all tracks (11.6 \pm 0.7$) \%$ of the digitizalions were lout because of the double-hit resolution, and the loss was about the same in all superlayers. For the leptons from the Higgs decay an average of $(7.3 \pm 0.6) \%$ of the digitizations were lost with the worst losses being in the inner superlayers.

\subsection{Pattern Recognition}

We began working on pattern recognition algorithms in order to examine our original design goals of finding track segments in auperlayers and removing bits from out-of-time bunch crossings. The algorithm for finding track segments was the following:

1. In each superlayer we identified "roads" containing hils. There are two parameters which can be varied: the width of the road and the number of hits required on the road. We used a width of five wires and required three or more hits out of eight possible. For ialated tracks one could require more hits; however, if two tracks are close together, as in fig. 5 , they will produce hits only on alternate layers and if one is lost due to the double-hit resolution there will be only three hits. The road requirement discriminates against low-pT tracks.

2. We required that at lcast one of the hits be in a layer with the opposite wire stagger from the others so that the left-right ambiguities could be resolved and hits from out-of-time bunch crossings rejected. 
3. We required that the hits be congistent with a straight line to within an error and in the process resolved the left-right ambiguitica.

Figure 8(a) shows all of the digitizations [11] for the event shown in fig. 6, including those from minimum bias background events. Figure 8(b) shows only those digitizations which are included in segunents. Keeping only thosi digitizations which form segments cleans up the events considerably. Figure $8(\mathrm{c})$ shows the tracks from the original event in the outer five superlayers in the region around the muon at the lower sight. Figure 8(d) dhows all of the digitizations in the event in the enlarged region (the digitizations are displayed at the locations of the hit wires). Finally, fig. 8(e) shows only those digitizations in the enlarged region which form track segments; here, the left-right ambiguities have been resolved, the drift times have been converted to distances, and the digitizations are displayed at the positions of closeal approach of the tracks to the wires. One can ciearly identify the muon track, and most of the extra hits have been removed.

Next, we applied our segment-finding algorithm to the $e$ and $\mu$ tracks from Iliggs boson decays. We defined two classea of segments: a "good" segment was one with at least five hits from a lepton track and no other hits, and an "OK" scgment was one with at least five hits from the lepton track and one hit from another track. The effects of hits from other tracks remain to be studied; we plan to compare measured nomenta wilh produced momenta in future work. With these definitions, we counted the number of segments found for each lepton track.

The distribution of the number of good segments for the e's and $\mu$ 's in the Higgs events is show in fig. $9(\mathrm{a})$. 'The corresponding distribution of total (good or OK) segments is shown in fig. 9(b). We sce that the lepton tracks from lliggs decay 
Jave an average of about 8 good segments and 10 total segments oul of 13 possible. Typically 30-50\% of segments were good in the inner superlayers, increasing to almost $80 \%$ for the outer superlayers. When $O K$ segments are counlext as well, $50.60 \%$ of segments are accepted for inner superlayers and over $80 \%$ for outer superlayers.

\subsection{Future Work}

We are planning to continue our tracking simulation studies using the software we have developed. Future work will include simulation of small-angle stereo wires and cathode strips for reconstruction of the direction along the wires; linking of seginents, both axial and stereo, to form tracks; studying how much additional information is needed from cathode strips to link the stereo segments properly; a more realistic simulation of electron drift in small-cell or straw tube drift chambers, including the effects of $\mathbf{E} \times \mathbf{B}$; and conceptual design and aimulation of intermediats tracking, as described briefly in sec. 2.3. In addition, we will study tracking for different physics processes, such as new heavy fermions, superaymmetric particles, and high-pr two-jet events, and begin to develop a realistic design for a tracking system for a complete SSC detector, including other detector componenls.

\section{CONCLUSIONS}

We have shown that an SSC tracking system design based on a pattern recogaition strategy of finding track segments in superlayers appears to provide a powerlul means of finding tracks in complex SSC events, even in an environment of inultiple events from several bunch crossings. So far, detailed simulations have verified the concepts developed over several years for SSC tracking delectors. An algorithm 
for finding track segments such as that described here could be used in the trigger for high-pr tracks. Depending on the effects on the plyssics analyses, we might envision making this requirener. at the processor level, reading out only the hits that form track segments. Although a great deal of werk remains to be done, we are hopeful that an SSC tracking system based on conventional wire chambers will enable us to explore the new physics which awaits us in the SSC regime.

\section{ACKNOWLEDGMENTS}

We would like to thank members of the SLD Collaboration at SLAC, especia "ly D. Aston, for their help in getting GEANT running on the SLAC IBM computer and for providing the interface to GEANT graphica. We gratefully acknowledge the support of the U. S. Department of Energy Program for Generic Detector Research and Development for the SSC. 


\section{AGFERINCXS}

[1] D. G. Casol, G. G. Hanoon et d., "Repert of the Centrd Tracting Group," in Procedings of the 1986 Stmmer Study os the Physics of the Saperconducling Stpercollider, Snowmase, CO, 1996, eds. R. Donaldoon and J. Marx, p. 377.

[2] G. G. Henson, S. Mori, L. G. Pondrom, H. H. William el d., "Report of the Iarge Solenoid Detector Group," in Pracezliags of the Workahep on Brperimente, Detectors, and Experimendel Arew for the Supercolliter, Berkeley, CA, 1987, edt. R. Donaldeon and M. G. D. Gilchrieac, p. 30.

[3] Rudiation Level in the SSC htersetion Regions, Takk Foroe Report, eds. D. E. Groom, SSC-SR-1033, SSC Central Deign Group, June 1988.

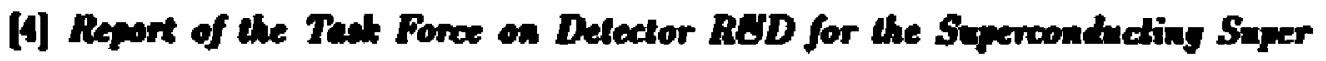
Collider, SSC-SR-1021, SSC Central Deigh Group, June, 1986, pp. 44-60; i. G. D. Gikhriese, in Procedinges of the 1984 Snmer Study on the Derign and Uilization of the Saperoonducting Swper Collider, Snowmat, CO, 1984, ed. A. Donaldson and J. G. Morfin, p. 607; G. Hanooa and D. Meyer, ibid., p. $\mathbf{5 8 5}$.

[5] J. V'vra, in Procedings of the Wortahop on Rediation Damage to Wire Chembers, Lawence Berkeley Latontory, Betieley, CA, 1986, od. J. Kadyk, p. 263.

[6] A. H. Waknta, Nucl. Instr. and Meth. 217 (1983) 65.

[7] R. L. Gluckstern, Nucl. Instr. and Meth. 24 (1963) 381. 
[8] F. E. Paige and S. D. Prolopopescu, "ISAJET 5.30: A Monte Carto Event Generator for $p p$ and $\bar{p} p$ Interactions," in Procedings of the 1986 Summer Study on the Physics of the Supenconducling Supencollider, Snowmas, CO, 1986, eds. R. Dondideon and J. Marx, p. 320. (The curnent verdion of ISAJET is 6.12)

[9] R. Brun, F. Bruyent and A. C. McPheroon, GaANTs Uarr' Guide, CeRN DD/EE/84-1.

[10] A. P. T. Paloumek, "Simulating a Central Drift Chamber for a Large Soletsid Detector at the SSC," SLAC-PUB-4787.

[11] The GEANT3 graphic does not diplay digitieation, only hits. To display digitications, they muat be converted to apatial coordinates and stored in the hil bonk. 
Table 1

Summary of Large Solenoid Detector Central Tracking System ([rom ref. (2))

\begin{tabular}{|c|c|c|c|c|c|c|}
\hline $\begin{array}{l}\text { Supetilayer } \\
\text { Number }\end{array}$ & $\begin{array}{l}\text { Inner } \\
\text { Rendius } \\
\text { (cm) }\end{array}$ & $\begin{array}{c}\text { Module } \\
\text { Thickness } \\
\text { (cm) }\end{array}$ & $\begin{array}{l}\text { Half } \\
\text { Length } \\
(\mathrm{cm})\end{array}$ & $\begin{array}{c}\text { Straw } \\
\text { Diameter } \\
\text { (mm) }\end{array}$ & $\begin{array}{c}\text { Rapidity } \\
\text { Range }\end{array}$ & $\begin{array}{c}\text { Cell } \\
\text { Occupancy } \\
(\%)\end{array}$ \\
\hline 1 & 40 & $2 . T$ & 85.2 & 3.92 & 1.50 & 12.1 \\
\hline 2 & 48 & 2.7 & 85.2 & 392 & 1.34 & 9.1 \\
\hline 3 & 56 & 2.7 & 119.0 & 3.92 & 1.50 & 8.8 \\
\hline 4 & 64 & 2.7 & 1190 & 3.92 & 1.38 & 7.0 \\
\hline 5 & 72 & 4.1 & 1190 & 5.89 & 1.28 & 13.0 \\
\hline 6 & 80 & 4.2 & 170.0 & 6.04 & 1.50 & 14.5 \\
\hline 7 & 88 & 4.2 & 170.0 & 6.17 & 1.41 & 12.9 \\
\hline 8 & 95 & 4.3 & 170.0 & 6.28 & 1.34 & 11.6 \\
\hline 9 & 104 & 4.4 & 170.0 & 6.38 & 1.27 & 10.5 \\
\hline 10 & 112 & 4.5 & 238.5 & 6.47 & 1.50 & 11.9 \\
\hline 11 & 120 & 4.5 & 238.5 & 6.55 & 1.44 & 10.9 \\
\hline 12 & 128 & 4.6 & 2985 & 6.61 & 1.38 & 10.0 \\
\hline 13 & 136 & 4.6 & $2 \times 8.5$ & 6.68 & 1.33 & 9.3 \\
\hline 14 & 144 & 4.6 & 228.5 & 6.73 & 1.28 & 8.5 \\
\hline 15 & 152 & 4.7 & 2285 & 6.78 & 1.23 & 7.9 \\
\hline
\end{tabular}




\section{PIGURE CAPTIONS}

Fig. 1. The charged particle flux and annual radiation dose as a function of perpendicular distance from the beam under atandard SSC operating conditions (from ref. [3]).

Fig. 2. Schematic vicw of the Large Solenoid Detector from the 1987 Berkeley Workstup [2].

Fig. 3. Layere of atraw tubes in a superlayer with every other layer ataggered by the stran tube radiua. A single in-time track will appear as a series of hits on the wires on alternate sides of the track. The left-right ambiguity is easily resolved locally. A track from an out-of-time bunch croasing will produce hile which are diuplaced trom ponsible tracks by at least $16 \mathrm{ng}$ in drift time.

Fig. 1. (a) Schematic viem of central and intermediate tracking ayatem in the Large Solenoid Detector. (b) Momentum resolution an a function of polar angle and repidity in the Large Solenoid Detector for the 13 auperlayers at radii $>50 \mathrm{~cm}$ in the central tracking rystem and including intermediate tractiog (from rel. [2]).

Fig. 5. (a) Two-jet event from ISAJET with $p_{T}>1 \mathrm{TeV} / \mathrm{c}$ in a 2-Tesla magnctic fidd in a detector of the geometry of the Large Solenoid Detector. There are 223 particles with $p_{T}>200 \mathrm{MeV} / \mathrm{c}$ and $|n|<1.5$. Converted photons and background from minimum bie events are not shown. (b) Enlargemer" of the event in the outer two superlayer in the ares of the dense jet at the lop of the deteclor. (c) Earlieat hit in each cell for the tracks shown in (b).

Fig. 6. Example of a Higfs event in the simulaled central tracking system. The leplons from the Higgs decay are indicaled by the heavier lines. Converted photons and other inleractions with the malerial are included. 
Fig. 7. (a) Total number of digitizations in Higgs events, including digitizations from minimum bias background events. (b) Fraction of digitizations from the minimum bias background events. The mean is $0.572 \pm 0.011$.

Fig. 8. (a) All of the digitizations for the lliggs event shown in fig. 6, including those from minimum bias background events. (b) Digitizations for this event which are included in track segments, as defined in the text. (c) Tracks from the original event in an enlarged region in the ouler five superlayere in the region around the muon at the lower right. (d) All of the digitizations in the event in the enlarged region of (c) (the digitizations ure displayed at the locations of the hit wires). (e) Only those digitizations in the enlarged region which form track segments. Here, the left-right ambiguities have been resolved, the drift times have been converted to distances, and the digitizations are displayed at the positions of closst approach of the tracks to the wires.

Fis. 9. (a) Distribution of the number of good segments ont of 13 possible for the e's and $\mu$ 's from the Higgs decays. (b) Diatribution of the number of tolal segments (good or OK) for the leptons from the Higgs decays. 


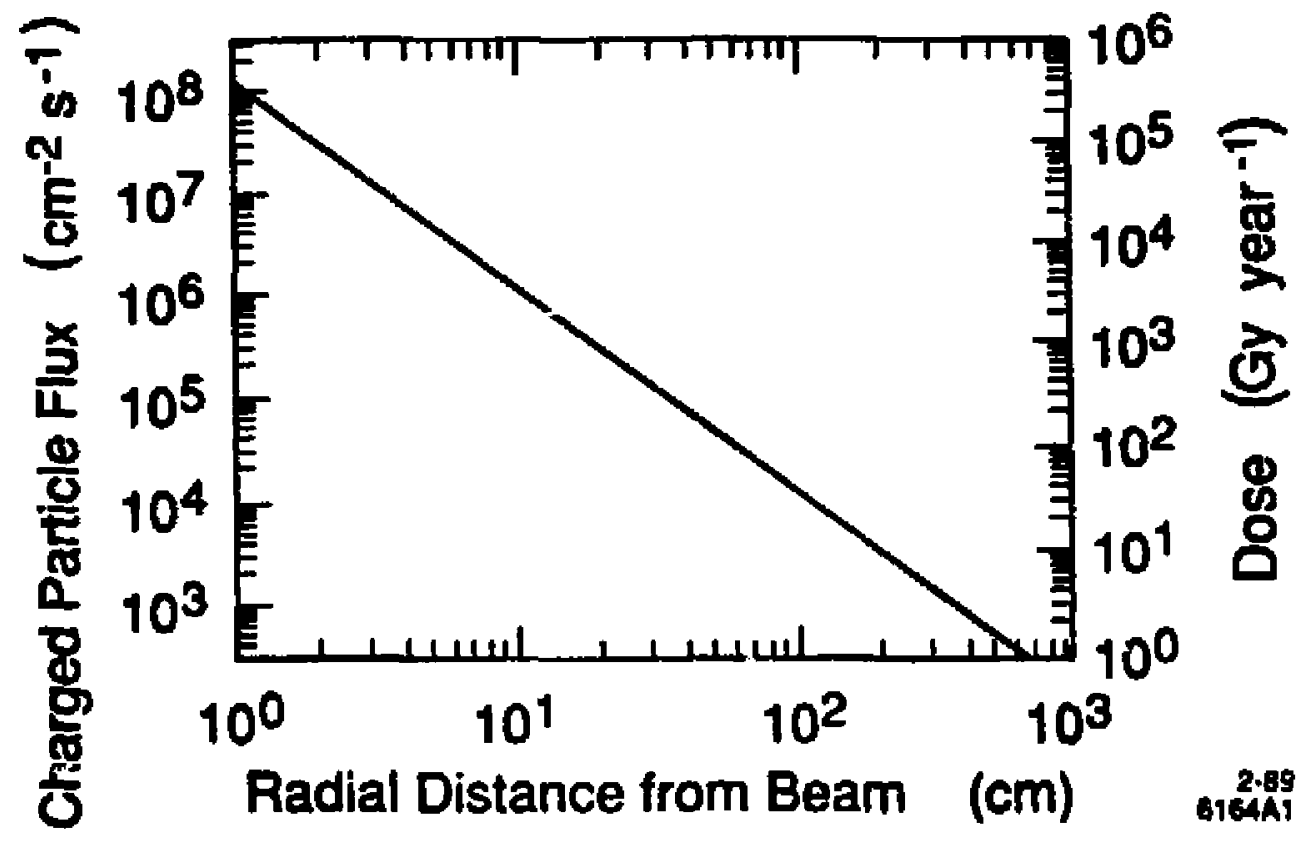

Fig. 1 


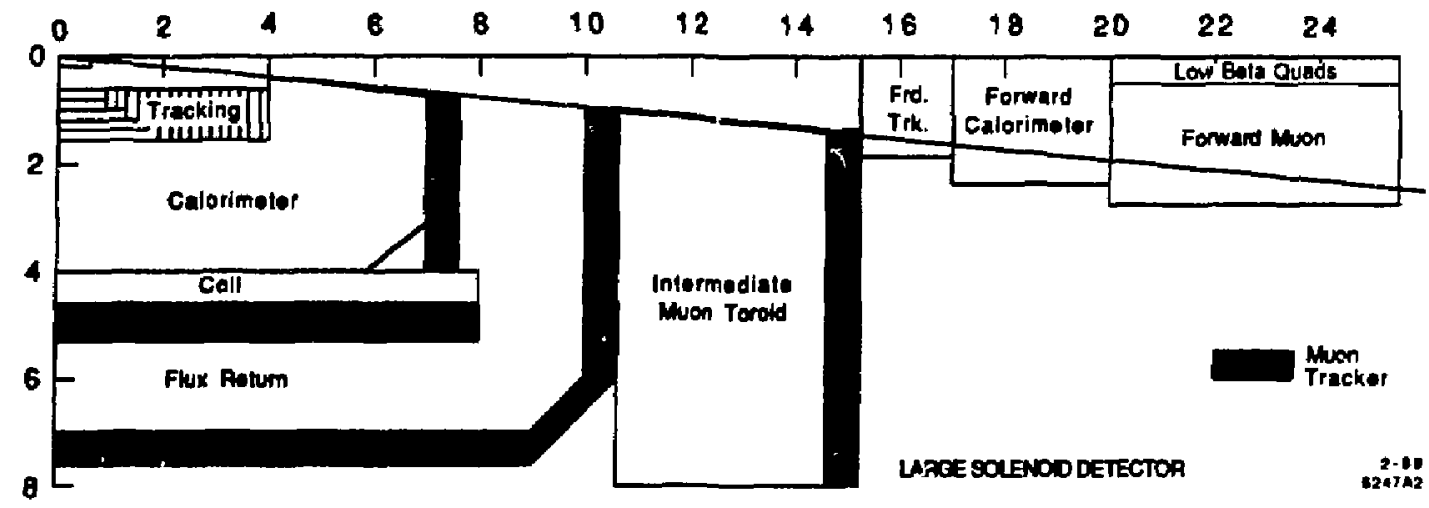

Fig. 2 
$x$ Sense Wire

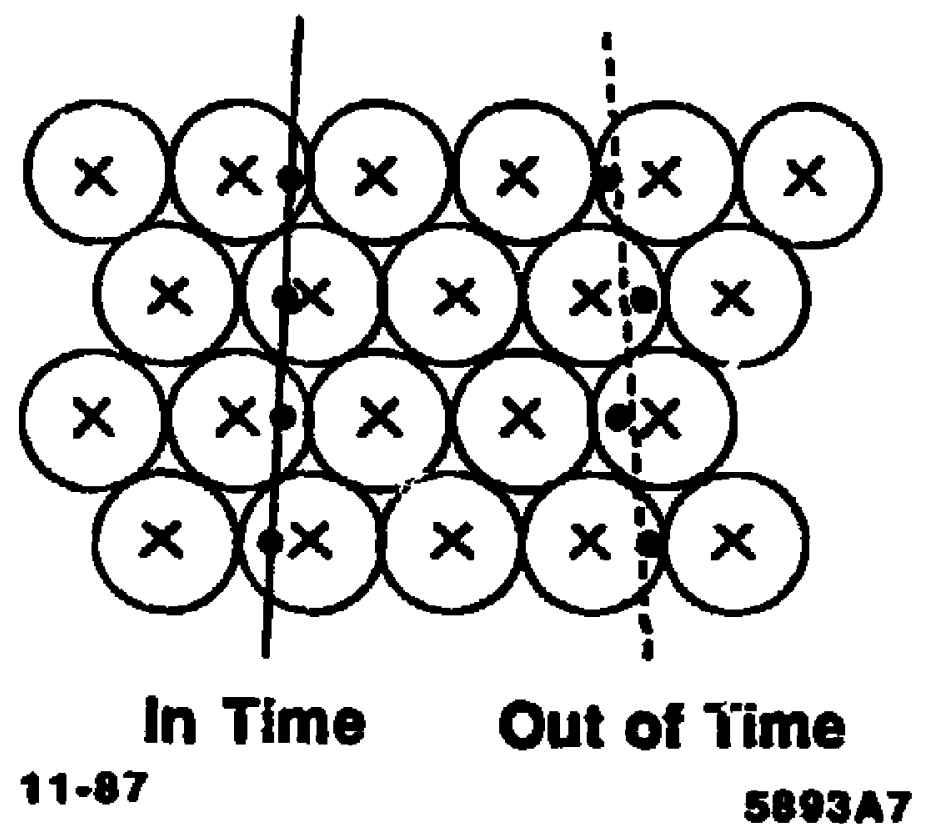

Fig. 3 


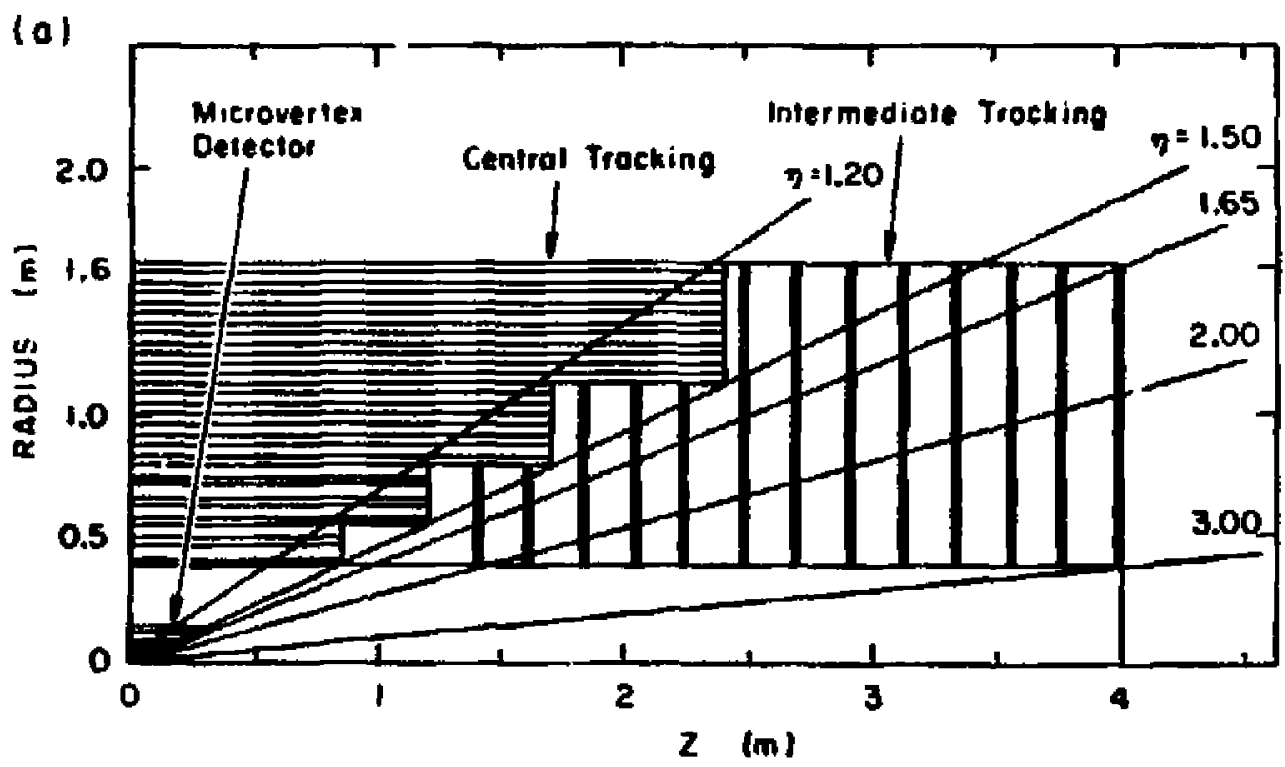

(b)
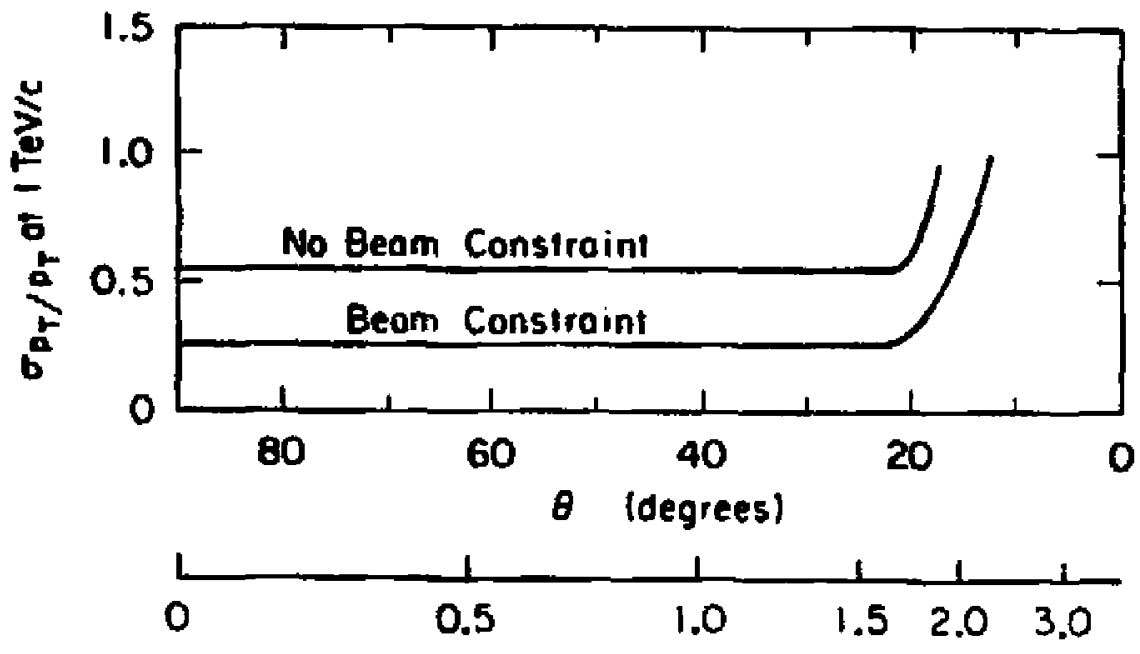

2-8s

$\eta$

624744

Fig. 4 

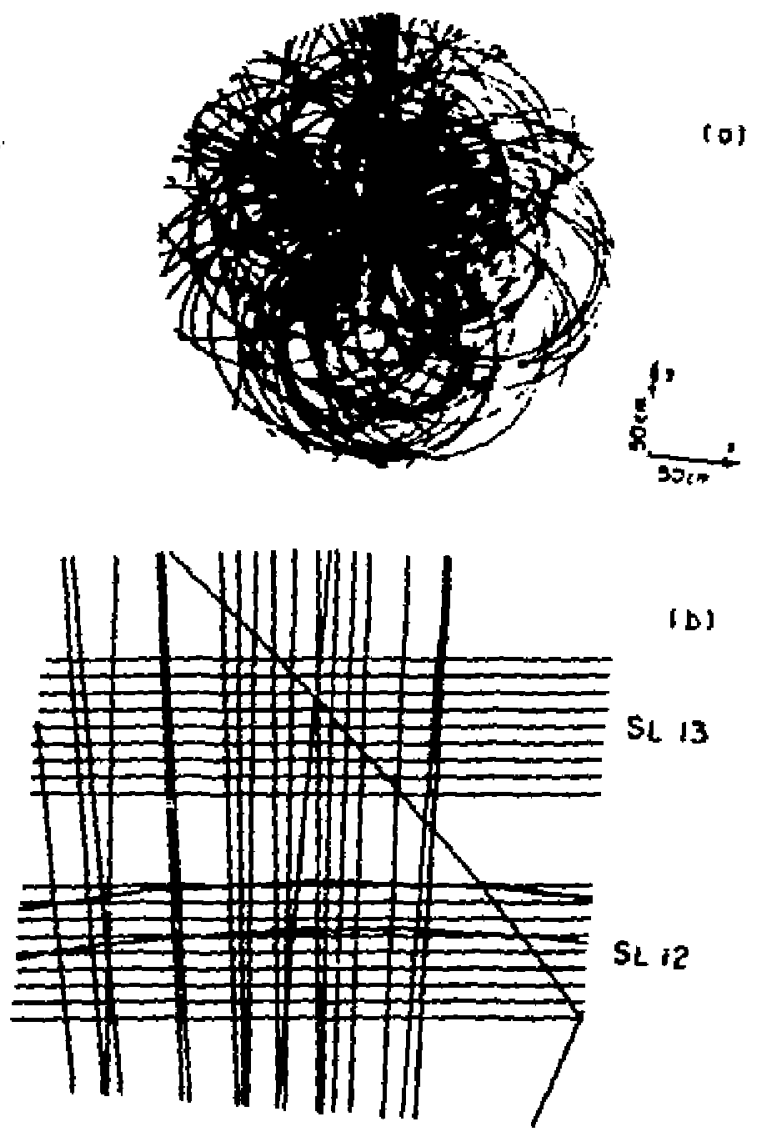

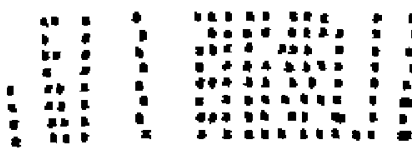

b)

\section{3}

(c)

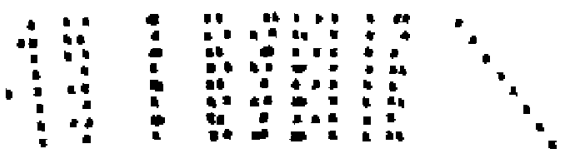

Eouless $\mathrm{H}$, in Cell Only

sh-ae

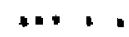

peater

Fig. 5 

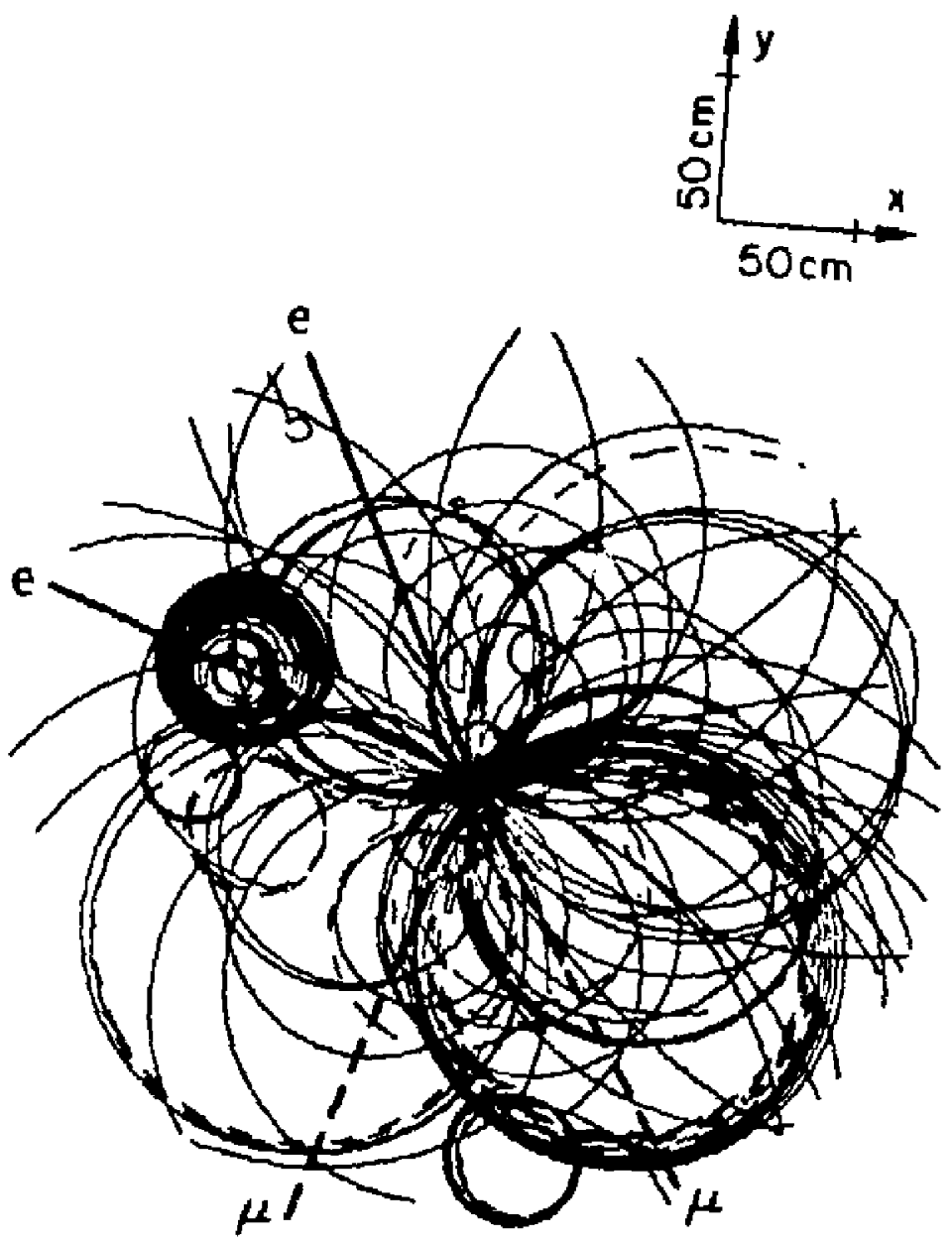

$11-88$

6164 A9

Fig. 6 

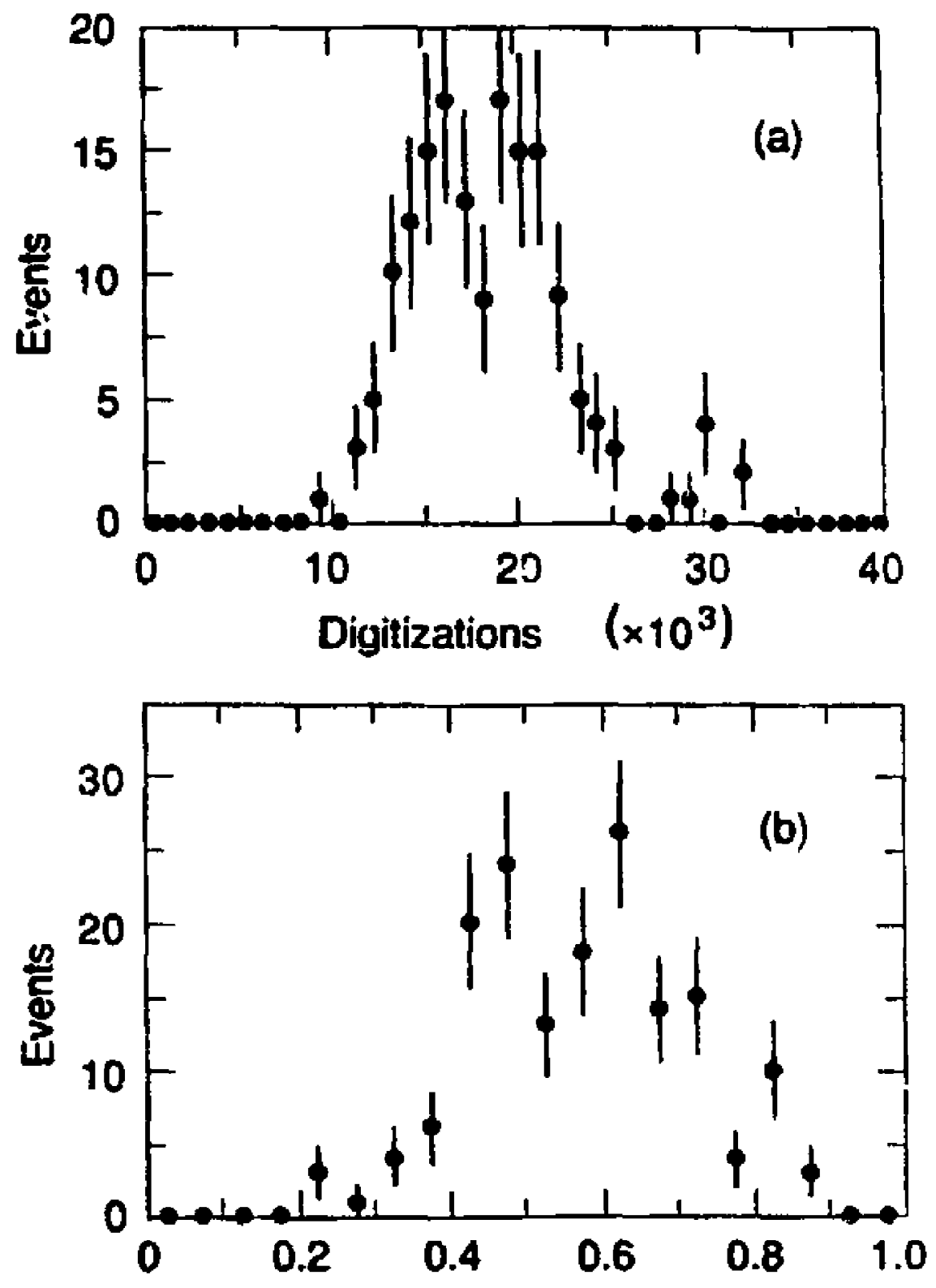

Fraction of Digitizations from Minimum Bias 
(o)

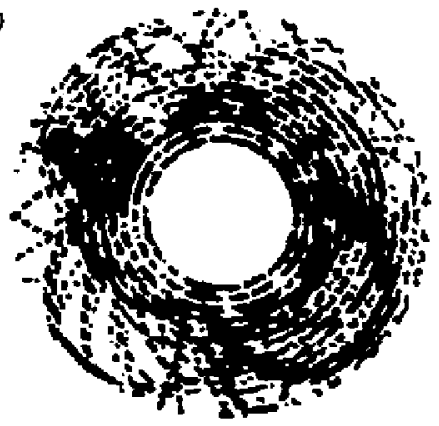

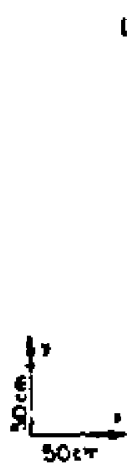

(b)
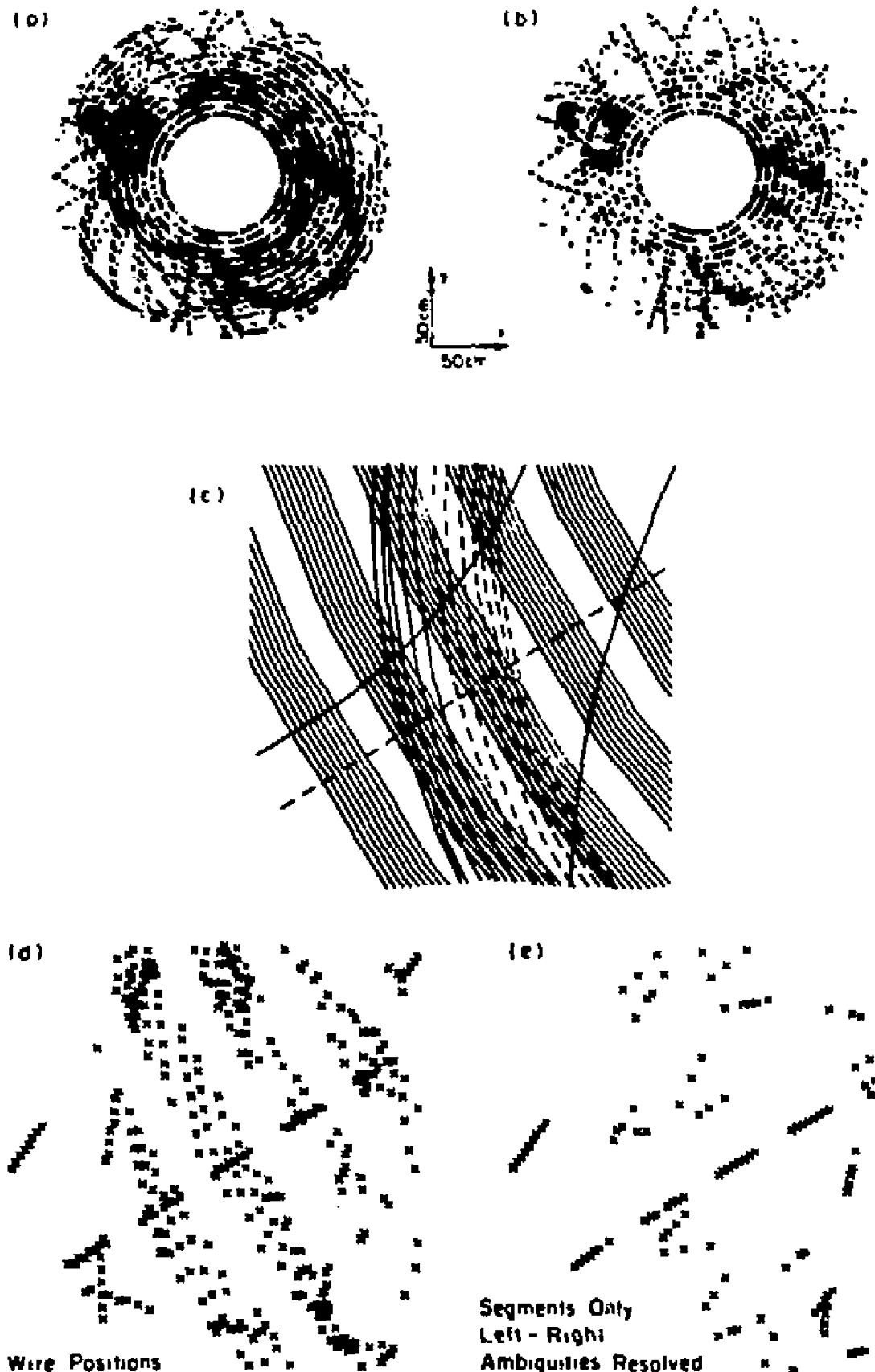

Disployed lor Hils

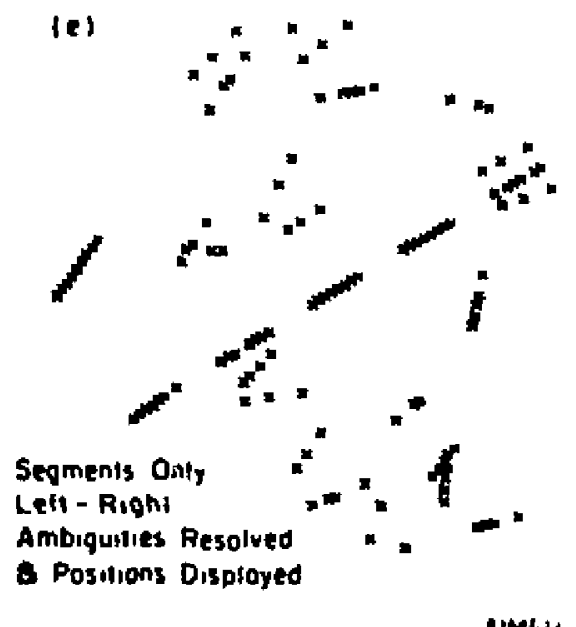

Fig. 8 


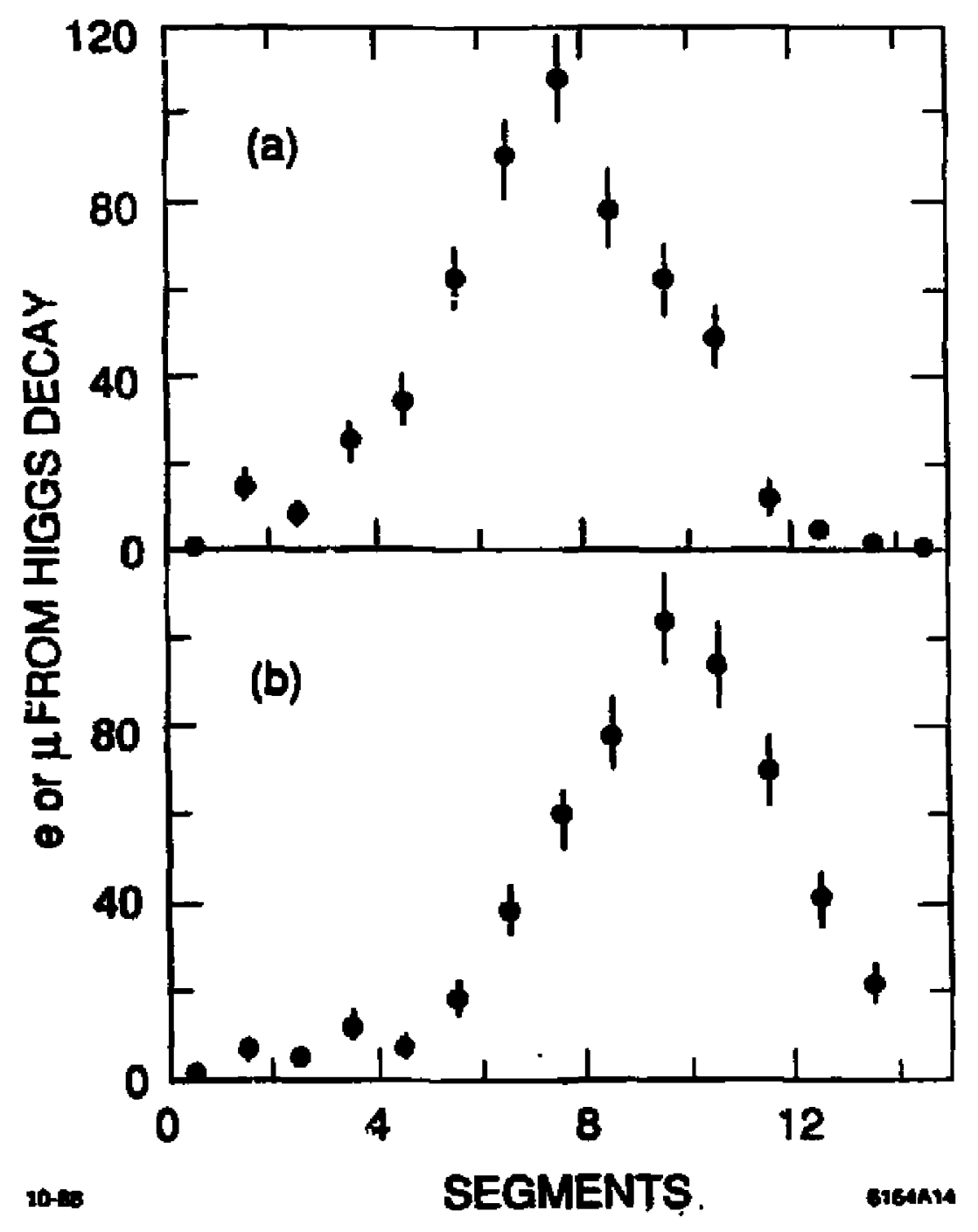

Fig. 9 


\section{DISCLAIMER}

This report was ptepared as an account of work sponsored by an agency of the United States Government. Neither the United States Government nor any agency thereof, nor any of their employees, makes any warranty, express or implied, or assumes any legal liability or responsibility for the accuracy, completeness, or usefulness of any informatiun, apparatus, product, or process disclosed, or represents that its use would not infringe privately owned rights. Reference herein to any spe ific commercial product, process, or service by trade name, trademark, manufacturer, or other vise does not necessarily constitute or imply its endorsemert, reconmendation, or favoring by the United States Goverament or any agency thereof. The views and opinions of authors expressed herein do not necessarily state or reflect those of the United Stales Government or any agency thereof. 